\title{
Mitochondrial Delivery of Biologically Active Molecules
}

\author{
Volkmar Weissig
}

Received: 13 September 2011 / Accepted: 13 September 2011 / Published online: 21 September 2011

(C) Springer Science+Business Media, LLC 2011

Starting in 1988 and throughout the 1990s, a series of key discoveries was made which revitalized scientific interest in mitochondria, a thoroughly studied cell organelle first described over a century ago. Long-time mitochondrial aficionados would argue that the 1990s just brought mitochondria back into fashion and thereby into more fashionable journals. Both views seem correct. The appearance of a textbook image of an electron micrograph of an animal mitochondrion on the front cover of Science on October 22, 1999 serves as verification, and Stephen Hersh's commentary in this theme issue properly reflects the excitement over this recent development.

During the 1990s, the number of human diseases found to be caused by defective mitochondrial DNA has grown dramatically, and nowadays approximately more than 360 mitochondrial disorders are known. It has been estimated that every $15 \mathrm{~min}$, a child is born who has a mitochondrial DNA disease or will develop one by the age of 5 (United Mitochondrial Disease Foundation, Pittsburgh, PA). Every organ at any age of onset can be affected. Also during the 1990s, mitochondria as the well-known "powerhouse" of the cell were accepted as the cell's "death switch" mirroring their recognized key role in programmed cell death (apoptosis). Consequently, around 1998, mitochondria were discussed for the first time as a novel potential target for cancer chemotherapy. "Talking cancer cells into committing suicide" by turning on the intracellular "death switch" emerged as an intriguing new idea in the war on cancer.

Further, mitochondria have been known as a source of free radicals for over 40 years, and the link between a

V. Weissig $(\bowtie)$

Midwestern University College of Pharmacy Glendale

Glendale, Arizona, USA

e-mail:vweiss@midwestern.edu variety of clinical conditions and an increased mitochondrial production of reactive oxygen species (ROS) under hypoxic conditions has been established. Subsequently, the idea of supplementing mitochondria with antioxidants always appeared as an attractive therapeutic approach to protect cells and tissues from oxidation. Only during the 1990s, however, the vulnerability of essential redox sensitive signaling pathways towards exogenous antioxidants was revealed, and the question of mitochondria-specific and, above all, controlled delivery of antioxidants arose. Finally, the key role mitochondria play for the calcium homeostasis of every cell and this organelle's involvement in almost every biochemical pathway has been textbook knowledge for perhaps half a century.

By the end of the 1990s, mitochondria had been established as an outstanding prime pharmacological target for an enormous variety of cytotoxic and cytoprotective therapies providing ample opportunities for drug development. Among pharmaceutical scientists mainly working in the area of drug delivery, however, interest in mitochondria appeared around 2000 as somewhat weak at best. Faulty notions, like once a drug is in the cytosol it will reach mitochondria anyway because there are so many of them in every cell, were not unheard of. Also, categorizing properly abstract submissions involving early mitochondrial drug delivery work to, for example, annual meetings of the American Society of Gene Therapy or the Controlled Release Society, during those years was somewhat hampered due to the lack of the term "mitochondria" in their official key word listings. To draw the attention of a broad audience in the drug delivery community to this fascinating cell organelle, Elsevier's Advanced Drug Delivery Reviews dedicated in 2001 an entire theme issue to "Drug and DNA Delivery to Mitochondria" (vol. 49, 2001, nos.1-2, V. Weissig, V.P. Torchilin, Eds.). It should not go unmen- 
tioned that it was quite challenging to adequately cover the "delivery" aspect therein.

Fast-forward to 2011-the scientific landscape has changed. During the last decade, sub-cellular, i.e. organelle-specific drug targeting, has become a new frontier for drug delivery. First, it has become evident that a large number of pharmacologically active molecules exert their activity on molecular targets at or even inside cell organelles. Any molecule which does not have the required organelle-specificity per se (based on its physico-chemical properties) would have to depend on a random, collisional interaction with its organellar target. Barring any organellespecificity of a potentially therapeutic drug, a targeting strategy for the intended subcellular compartment would need to be devised in order to potentiate therapeutic effect. Moreover, in the case of molecules with a stronger affinity for a non-target subcellular compartment, an even greater need exists for the ability to control the subcellular distribution of that molecule. Second, the emergence of nanoscience and with it the creation of nanomaterials with unprecedented properties has had and most certainly is still having a significant impact on setting the quest for subcellular drug delivery into motion.

During the last ten years, an increasing number of laboratories have started designing low-molecular-weight compounds with physico-chemical properties suited for mitochondrial accumulation. Other groups began developing pharmaceutical nanocarrier systems for the targeted delivery of all sorts of biologically active molecules to/into mitochondria. The overarching goal is to selectively manipulate and/or probe mitochondrial functions under physiologic as well as pathologic conditions.

This theme issue is dedicated to a large variety of aspects of Mitochondrial Pharmaceutics and Mitochondrial Medicine. One group of articles deals with the improvement of established and creation of new means to deliver biologically active molecules to and into mitochondria. Netanel Kolevzon et al. in Eylon Yavin's laboratory (Hebrew University of Jerusalem) describe a synthetic methodology for the delivery of a biologically active peptide into mitochondria of intact cells by means of attaching multiple triphenyl phosphonium cations to it. The potential of using a mitochondrial fraction isolated from cells to prepare liposomes capable of delivering biologically active molecules to mitochondria of live mammalian cells is demonstrated by Maryura Wagle and Laura Martinville in Gerard D'Souza's laboratory at the Massachusetts College of Pharmacy and Health in Boston. Iris Geisler and Jean Chmielewski (Purdue University) report a significant increase of cellular uptake and mitochondrial localization of cell-penetrating peptides following peptide dimerization. Kelly Stewart and Rida Mourtada in Shana O. Kelley's laboratory (University of Toronto) describe the creation of a new class of mitochondria-penetrating peptides with delocalized lipophilic cations inserted into the peptide sequence. A comprehensive review of the transduction of human recombinant proteins into mitochondria is provided by Lefkothea Papadopoulou and Asterios Tsiftsoglou (Aristotle University of Thessaloniki). Nunzio Denora et al. from Giuseppe Trapani's laboratory (Universita' degli Studi di Bari) describe the utilization of new fluorescent probes targeted at a mitochondrial-located translocator protein for imaging activated microglia. The impact of a drug's physico-chemical properties on its delivery to brain mitochondria is assessed by Shelly Durazo et al. in Uday Kompella's laboratory (University of Colorado Denver). Finally, I discuss in my own review the historical development of mitochondria-targeted pharmaceutical nanocarriers and outline their manifold potential applications.

Another group of papers is dedicated to the topic of manipulating the mitochondrial genome. Diana Lyrawati, Alan Trounson, and David Cram (Monash University) report the expression of GFP inside the mitochondrial matrix using DQAsome-mediated delivery of an artificial mini-mitochondrial genome. Brian Bigger and Charles Coutelle (Imperial College London) describe the stable cloning of the entire human mitochondrial genome in yeast. Noha Ibrahim et al. from Andre Dietrich's laboratory (University of Strasbourg) provide critical information for the optimization of DNA delivery into mitochondria, opening the prospect of targeting whole mitochondrial genomes into mammalian mitochondria.

Other papers focus on the role of mitochondria and antioxidants for the redox status of a cell. Marvin Edeas, the President of the International Society of Antioxidants in Nutrition and Health (ISANH), reviews current strategies for alleviating oxidative stress by administering antioxidants and analyzes potential causes for the widely observed ineffectiveness of clinical trials involving antioxidant therapies. The original discovery and the preclinical and clinical development of a novel class of small peptides that selectively target the inner mitochondrial membrane and protect mitochondrial function are reviewed by Hazel $\mathrm{H}$. Szeto (Cornell University) and Peter W. Schiller (Clinical Research Institute of Montreal). A series of dietary ingredients and metabolites able to activate endogenous antioxidant enzymes by initially inducing mitochondrial and cytosolic ROS production is described and termed "hormetics" by Marc Birringer from the Fulda University of Applied Science. Konstantin Lyamzaev from Boris Chernyak's and Vladimir Skulachev's laboratories at the Lomonosov Moscow State University describes plastoquinones conjugated with cationic plant alkaloids as novel mitochondria-targeted antioxidants. An acetate prodrug of a pyridinol-based vitamin $\mathrm{E}$ analogue with improved antioxidant activity is introduced by Omar Khdour and 
Jun Lu from Sidney Hecht's laboratory at Arizona State University. Nadezda Apostolova et al. from Victor M. Victor's group at the University of Valencia report about mitochondrial antioxidants alleviating oxidative and nitrosative stress in a cellular model of sepsis. The role of inhibitors of the succinate:quinine reductase/complex II for regulating mitochondrial ROS production is reviewed by Stephen Ralph et al. (Griffith University). Lynlee Lin et al. from Tarl W. Prow's laboratory at the University of Queensland describe the utilization of time-correlated single photon counting (TCSPC) for simultaneous, realtime quantification of $\mathrm{ZnO}$ nanoparticles and $\mathrm{NAD}(\mathrm{P}) \mathrm{H}$. The same laboratory in collaboration with Marc Schneider's laboratory from the University of Saarland reports about the impact of gold nanoparticle penetration into human skin on cell metabolism.

Differences in the metabolism between transformed and non-transformed cells are discussed by Emma Ramsey et al. from Pierre Dilda's laboratory at the University of New South Wales, focusing in particular on utilizing mitochondrial metabolic changes of tumor cells for selectively delivering drugs to cancer cells. The state-of-the-art technology using protein-based sensors for quantitative glucose and ATP sensing in living mammalian cells is reviewed by Dania Liemburg-Apers et al. from Werner Koopman's laboratory at the Radboud University Nijmegen Medical Center. Jean Soustiel et al. (Israel Institute of Technology) describe the effect a ligand for a mitochondrial translocator protein has on the reduction of mitochondrial membrane permeabilization under conditions of traumatic cerebral edema and metabolic crisis. Lianet Monzote from the Institute of Tropical Medicine in Havana reviews potential mitochondrial targets and drugs against Trypanosoma and Leishmania parasites as well as the role of mitochondria in the development of drug resistance.

Considering the current momentum and the wide variety of mitochondrial research as documented in this theme issue, it appears safe to assume that novel mitochondria-targeted therapies will become feasible in the near future.

\section{INTERVIEW WITH DR. VOLKMAR WEISSIG}

What do you think holds the key to your success as a pharmaceutical/ drug delivery scientist?

My interest in Life Science was without a doubt ignited during my secondary school years in Germany. We had an excellent educational program in East Germany that focused on individual scientific disciplines starting in the $5^{\text {th }}$ grade with Biology, followed by Physics in the $6^{\text {th }}$ grade, and Chemistry in the $7^{\text {th }}$ grade. After the $10^{\text {th }}$ grade, I was accepted into a special program hosted by the College of Chemistry in Leuna-Merseburg ("Spezialschule fuer Chemie"). Within two years, we mastered the curriculum of the $11^{\text {th }}$ and $12^{\text {th }}$ grades of High School ("Abiturstufe") and, at the same time, the whole curriculum of the $1^{\text {st }}$ year of the College's Chemistry program. These tough two years taught me studying techniques I am still utilizing today. We were not only taught by excellent High School teachers, but also already by dedicated College faculty. Later on, following my master thesis involving the synthesis of small antioxidant molecules, I had to switch several times my field of research, which I originally did not like but, in hindsight, I believe was very beneficial for my development. Repeatedly, I was forced to start a new project from scratch, to work myself into scientific sub-disciplines starting at the beginner's level, to learn new laboratory techniques I hadn't heard of before. I believe that the very broad scientific and experimental, methodological background I acquired during those years have helped me to be where I am today.

\section{What do you consider to be your key research accomplishments?}

During the last 15 years, I and my graduate students have pioneered the design and development of mitochondriatargeted pharmaceutical nano carriers. We have demonstrated their usefulness for the targeted delivery of DNA to mitochondria in living mammalian cells, thereby opening a new strategy towards mitochondrial gene therapy. We have provided evidence that the selective delivery of small proapoptotic molecules to mitochondria significantly increases their in vitro and in vivo efficacy as anticancer drugs, thus opening a new strategy in the battle against cancer. Overall, I consider having contributed during the last decade to making the idea of organelle-specific drug targeting widely accepted is one of my key research accomplishments.

\section{What was the turning point in your career?}

At the beginning of the 1990s, I switched yet another time my field of research. I stopped working with liposomes and entered the field of Parasitology. I am still grateful to Dr. Thomas C. Rowe (University of Florida, Gainesville, FL) for having accepted me as a Postdoc knowing that I never did any Southern, Western, or Northern blotting, never screened any library, never did any cloning and PCR, that my experience in Molecular Biology techniques was essentially zero. He asked me to study the impact of molecules known to interact with mitochondrial DNA on the DNA metabolism of Plasmodium falciparum in order to perhaps find new leads for the development of antimalarial drugs. Among the large number of compounds I had to 
screen for their ability to inhibit mitochondrial topoisomerases was dequalinium chloride, which turned out to become the biggest turning point in my career. Having the background of a liposomologist, I saw that dequalinium chloride seems to be able to self assemble into liposome-like vesicles following probe sonication of an aqueous suspension. Without planning on it, I made cationic vesicles completely composed of molecules known to specifically accumulate in mitochondria. Couldn't these vesicles be used to deliver DNA or any other molecules selectively to mitochondria? This idea never lost its grip on me, and in my "spare time" (if Postdocs have any such time to begin with), I started working towards this new goal. Since then, I have never switched my field.

\section{Which individuals most influenced your research career?}

Juergen Lasch from the Martin-Luther University in Halle, Germany, introduced me back in the early 1980s, to Liposome Technology. Due to his strong collaboration at that time with Vladimir P. Torchilin in Moscow, Russia, I was able to visit Dr. Torchilin's laboratory on several occasions. There, I learned a lot from Alexander (Sascha) Klibanov. Among my most memorable times were the three months I spent in 1988 in Gregory Gregoriadis's laboratory at the Royal Free Hospital in London, where I enjoyed working side-by-side with Judith Senior. This time was even more exciting for me, since it was the first time I could work in a laboratory beyond the "Iron Curtain." The fact that I could order in the evening a needed chemical from Sigma and get it on my bench the very next day seemed like a miracle to me. It sounds unbelievable nowadays, but in East Germany chemicals had to be ordered one year ahead of time. Therefore, often the direction of one's research was not determined by what scientific question one wants to answer, but what questions could be answered with the chemicals already sitting on the shelf. I am also grateful to Hans Schreier, who offered me in 1991 a Postdoctoral position at the University of Florida in Gainesville and, in so doing, opened my way into this great country I am still living in. Finally, and I referred to it already above, Tom Rowe's decision at the University of Florida to "let me play" with dequalinium chloride undoubtedly had the biggest influence on my more recent career.

Pharmaceutical scientists are faced with the dilemma of having to publish in biomedical or basic science journals. Does this mean cutting-edge science will not likely be featured in journals like Pharmaceutical Research?

The dilemma of having to publish in biomedical or basic science journals is obviously caused by the generally higher impact factors these journals possess, and I have two big concerns in this regard. First, as I see it, impact factors of journals depend to a certain extent on the size of their audience. New journals have emerged during the last decade having the term "nano" in their title, and these journals bypassed within a few years long-standing and established journals dedicated to a specific scientific discipline like Pharmaceutical Sciences. The reason for that, I believe, lies in the large variety of scientific disciplines covered by the overarching meaning of "nano." I cannot understand why, for example, articles about the physics and material properties of nano wires and articles about nano lipid vesicles (formerly called liposomes) should appear in the same journal. My second concern, can young scientists (and older alike) ignore the reign of the impact factor? Maybe yes, but only at one's own peril. At academic institutions, in my personal opinion, an unhealthy climate has evolved in which one's creativity and productivity is only measured by impact factors and dollars. Consequently, and to answer the above question, I tend to believe that "cutting-edge science" will indeed not likely be featured in journals like Pharmaceutical Research, which I consider a very unfortunate development. However, to contradict my own statement to some extent, I actually consider several articles in the theme issue presented here as cutting-edge research. For example, mitochondria-targeted peptides on the brink to Phase 2 clinical trials (Szeto et al.) as well as reports about the cloning of the "unclonable" human mitochondrial genome (Bigger et al.), and, in my opinion most importantly, the report about the first expression of an artificial minimitochondrial genome inside mitochondria in living mammalian cells (Lyrawati et al.) do represent cutting-edge research. I am very grateful to these authors for having accepted my invitation to submit their work to this theme issue of Pharmaceutical Research.

Where is the field of mitochondrial delivery of biologically active molecules going, and how do the articles in this theme issue fill the gap?

The eventual cure of mitochondrial DNA diseases, the launch of effective alternative anticancer therapies, the ability to control the redox status of a cell, as well as the design of new tools for probing mitochondrial functions I consider as major destinations the field of mitochondrial drug delivery is moving towards. Several articles in this theme issues are dedicated to each one of these four major goals.

What are the challenges for developing and commercializing mitochondrial delivery of biologically active molecules, and how can they be overcome?

Having been involved years ago in several attempts to commercialize mitochondria-targeted pharmaceutical nano carriers, I believe in hindsight the field just wasn't mature 
enough. Euphoric ideas, unfortunately, were not yet matched by hard data. At the same time, the general awareness of, for example, mitochondrial DNA diseases or the role mitochondria play in apoptosis, thereby controlling any life or death decisions of a cell, was and perhaps still is underdeveloped. Only in recent years have major funding organizations started to acknowledge the potential impact pharmaceutical research focusing on mitochondria will have on public health. Major funding will beget major new data plus intellectual property, which in turn will give rise to new start-ups, which eventually will gain the attention of Big Pharma. Furthermore, non-profit organizations like the United Mitochondrial Disease Foundation (UMDF, Pittsburgh, USA) and similar organizations in Europe and Asia are undertaking tremendous efforts to raise the public awareness of mitochondrial disorders. I am therefore very optimistic with respect to future commercialization of mitochondrial-targeted technology.

\section{What are the challenges facing drug delivery research and development?}

Enormous progress in Human Genomics/Molecular Biology has revealed an unprecedented number of potentially new therapeutic targets. Alongside with that, the creation of new chemical synthetic methods such as combinatorial chemistry has made the synthesis and identification of new drug candidates more efficient than ever before. To translate any potential new drug molecule into the clinic, it has to be formulated into specific dosage forms, and the crucial role formulation scientists play in the process is undisputed. Likewise, the continuous development and modernization of drug delivery devices for all different routes of administration has a long history in Pharmaceutical Science and is strongly fostered by Big Pharma. In contrast, however, the design and development of new "post-liposomal" pharmaceutical nano carriers or particulate drug delivery systems still seems to be the realm of academic laboratories or start-up companies at best. I believe it is the big challenge to scientists working in the general area of nano formulations/nano pharmaceuticals to translate the big promise Nanoscience is offering to Medicine into experimental data which will attract the interest and investment of Big Pharma.

What is the key to developing successful collaborative relationships between academic drug delivery scientists like you and more applied pharmaceutical/formulation scientists who can help in product development?

It certainly starts with the drug delivery scientist working in academia (like myself) having to develop technology which could potentially raise the interest of the more applied formulation scientists and, above all, of the company employing the latter. Both sides have to be convinced of the promise such new technology might have. To reach this initial point requires not only talking to each other, but even more educating each other about each other's quite different point of views. Having an open mind and being unbiased are essential for such preliminary talks. The "rest" should be easy. Trusting and respecting each other's wealth of expertise and experience in respective fields and sharing enthusiasm about the common goal of eventually commercializing a particular drug delivery technology are keys and the basis for any such successful collaborative relationship.

What is your philosophy of educating graduate students in your laboratory?

Though being their academic advisor, mentor, and teacher, I always consider graduate students as equal partners in tackling a scientific project. From early on I strongly encourage their independence and try to refrain from micromanaging their experimental work. Adapting George Patton's wisdom, I have always told my graduate students where to go but not how to get there, and I have never failed to be amazed by their ingenuity.

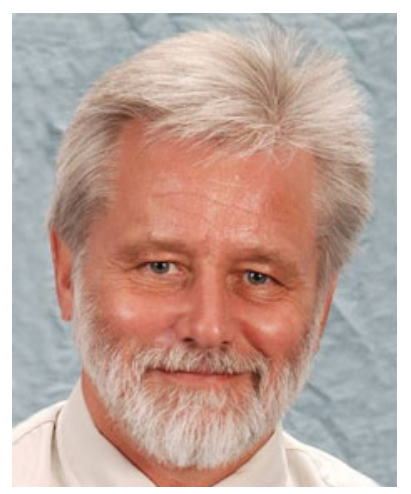

Dr. Volkmar Weissig is Professor of Pharmacology and Interim Chair of the Department of Pharmaceutical Sciences at Midwestern University College of Pharmacy Glendale in Glendale, Arizona, USA. He received his B.S., M.S., and Ph.D. degrees in Chemistry and his postdoctoral Sc.D. degree (the German "Habilitation") in Biochemistry and Pharmaceutical Biotechnology from the Martin-Luther University in Halle (Germany). Combined he completed several years of postdoctoral fellowships at the Cardiology Research Center in Moscow (Russia); the Academic Department of Medicine at the Royal Free Hospital School of Medicine in London (UK); the Institute of Organic Chemistry at the Czechoslovakian Academy of Science in Prague (CSFR); the College of Pharmacy and the College of Medicine at the University of Florida in Gainesville, FL, and at Harvard Medical School and Massachusetts General Hospital in Boston, MA. Before joining the faculty at Midwestern University, he was an Assistant Professor of Pharmaceutical Sciences at Northeastern University in Boston, MA. Since the beginning of the 1980s, Dr. Weissig has been working in the field of Liposome Technology. Toward the end of the 1990s, he turned his focus on Mitochondrial Pharmaceutics, an area he actively has been pioneering for the last 10 years.

Dr. Weissig holds 16 patents and has published over 80 research papers, review articles and book chapters, mostly in the area of nano drug delivery systems. Several of his book chapters have been translated into Chinese, German, and Polish. He has also edited four books. He serves as the Associate Editor of the Journal of Liposome Research and is a member of several other Editorial Boards. In 2004, 
Dr. Weissig was elected as the Vice Chair and the following year Chair of the American Association of Pharmaceutical Sciences Northeast Regional Discussion Group (AAPS-NERDG). He also is an active member of the International Liposome Society, Mitochondrial Research Society, American Association of Pharmaceutical Sciences, and American Society for Advancement of Science. In
2002, Dr. Weissig was elected as member of the Rho Chi Honor Society Beta Tau Chapter at Northeastern University in Boston, MA, and in 2011 he was inducted into the Kappa Psi Pharmaceutical Fraternity Delta Sigma Chapter at Midwestern University. In July 2009, Dr. Weissig was elected as a Fellow of the World Technology Network. 